Basic Health Sciences

Poster

Abstract ID: 19

\title{
Investigation of an antidiabetic potential of a phenolic compound isolated from the leaves of mempelas paya
}

\author{
Qamar Uddin Ahmed | Siti Zaiton Mat Soad | Mehnaz Afrin | Wan Mohd. Azizi Wan Sulaiman \\ Kulliyyah of Pharmacy, International Islamic University Malaysia
}

Introduction: Leaves of mempelas paya (Tetracera indica) (family: Dilleniaceae) is traditionally used in diabetes management in Malaysia. However, no attempt has ever been made to discover an active principle responsible for its blood glucose lowering effect. Hence, this research deals with the isolation, in vivo antidiabetic and toxicological evaluation of an active principle. Methods: Chemical investigation of methanol extract derived from the powdered leaves resulted in the isolation of a phenolic compound through repeated silica gel column chromatography. The structure was elucidated by spectroscopic (1H-NMR, 13C-NMR, MS, IR and UV) analysis. In vivo antidiabetic and toxicological studies were carried out using Sprague-Dawley rats (diabetic and normal) at three different concentrations (1, 5 and $25 \mathrm{mg} / \mathrm{kg}$ b.w.). Results: 5,7-dihydroxy-8methoxyflavone was found to exhibit significant anti-hyperglycaemic activity $(p<0.05)$ in diabetic rats at 5 and $25 \mathrm{mg} / \mathrm{kg}$ b.w. without showing hypoglycaemic effect in normal rats. The anti-hyperglycaemic activity was found to be comparable with glibenclamide (GLBC) $(\mathrm{p}<0.05)$. In vivo toxicological study for continuous 21 days showed no abnormal activity and mortality. Histopathology of kidney, liver and pancreas of both normal and diabetic treated rats demonstrated normal and improved condition when compared to diabetic control group. There were no significant changes seen in the kidney (serum urea and creatinine) and lipid (TC, TG, LDL-C and HDL-C) functional parameters tested. Conclusions: 5,7-dihydroxy-8-methoxyflavone isolated from the leaves of mempelas paya coud be a safe antidiabetic agent.

KEYWORDS: Tetracera indica, 5,7-dihydroxy-8-methoxyflavone, in vivo, antidiabetic activity, toxicological study, histopathology 